\title{
On the possible presence of promethium in the spectra of HD 101065 (Przybylski's star) and HD 965^
}

\author{
C. R. Cowley ${ }^{1}$, W. P. Bidelman ${ }^{2}$, S. Hubrig ${ }^{3}$, G. Mathys ${ }^{3}$, and D. J. Bord ${ }^{4}$ \\ 1 Department of Astronomy, University of Michigan, Ann Arbor, MI 48109-1010, USA \\ 2 Department of Astronomy, Case Western Reserve University, Cleveland, OH 44106-7215, USA \\ 3 European Southern Observatory, Casilla 19001, Santiago 19, Chile \\ e-mail: [shubrig; gmathys] @eso.org \\ 4 Department of Natural Sciences, University of Michigan-Dearborn, Dearborn, MI 48128-1491, USA \\ e-mail: dbord@umd.umich.edu
}

Received 21 November 2003 / Accepted 23 February 2004

\begin{abstract}
Traditional and statistical line-identification methods indicate the presence of Pm I and II, Tc I, and perhaps Tc II in the spectrum of the roAp star HD 101065. These methods also lead to the presence of Pm II and probably also Pm I in a related cool Ap star, HD 965. The spectroscopic evidence is strong enough that we would declare promethium to be present without hesitation, if any of its isotopes were stable. The longest-lived promethium isotope has a half-life of only 17.7 years. The presence of this element would mean that unrecognized processes - perhaps flare activities - are taking place in the atmospheres of these stars. The significance of such processes for galactic chemical evolution cannot be ruled out. We discuss the possibility that the highly improbable wavelength coincidences are due to chance, or due to contamination of the laboratory sources.
\end{abstract}

Key words. stars: chemically peculiar - stars: abundances - stars: activity - stars: atmospheres - methods: data analysis

\section{Introduction}

The literature of the last half of the 20th century contains several attempts to identify elements with half-lives significantly shorter than a billion years (see Jaschek \& Jaschek 1995). Of these, only that of Tc I in peculiar red giants has been generally accepted.

We present evidence here that lines of Pm I and/or II are present in the spectrum of HD $101065\left(=\mathrm{CoD}-46^{\circ} 5445\right.$, also known as Przybylski's star). The first, and possibly also the second spectrum of technetium may also be present. The highly peculiar spectrum of this star is well known to stellar astronomers. It is believed to be an extreme member of a class of stars whose surface chemical peculiarities are generally thought to be a consequence of chemical separation. This theory alone, however, would not account for the presence of elements with no long-lived stable isotopes. We have found that an even stronger case for the presence of Pm I and II could be made for the "Przybylski twin" (Hubrig et al. 2002),

Send offprint requests to: C. R. Cowley,

e-mail: cowley@umich.edu

* Based on observations collected at the European Southern Observatory, La Silla (Chile), as part of programme No. 60.E-0564, and Paranal (Chile), as part of programmes Nos. 68.D-0254 and 70.D-0470.
HD $965\left(=\mathrm{BD}-0^{\circ} 21\right)$. We discuss material for this star after covering HD 101065.

In the 1960's, it seemed possible that surface nuclear reactions played a significant role in creating the abundance anomalies of upper main sequence stars (cf. Fowler et al. 1965). These ideas were completely abandoned in favor of Michaud's (1970) far more plausible theory of chemical separation by slow diffusion currents.

In the same year as Michaud's classic paper, Aller \& Cowley (1970) suggested the "possible presence of promethium" in the spectrum of the highly peculiar Ap star HR 465 (=HD 9996). The putative Pm II identification was based on spectra obtained by one of us (WPB) at Lick Observatory in the early 1960's. His spectra and extensive line identification list revealed the most complicated spectra of all Ap stars - with the possible exception of HD 101065.

Preston \& Wolff (1970) showed that HR 465 was a spectrum variable with a period of 22-24 years. In 1970, the spectrum was not particularly unusual for an Ap star. Indeed, none of the spectra studied by Preston \& Wolff showed the extreme rare-earth strengths seen on the Lick spectra. Thus, the Pm II identification could neither be confirmed nor refuted on the basis of new data. After some speculations, criticism, and rebuttal in the early 1970's, the matter has not been revisited. Extensive surveys of high-dispersion spectra from the Dominion Astrophysical Observatory were made in the 
1970's (cf. Cowley 1976, 1984). These studies incorporated the computer-automated method of wavelength coincidence statistics (WCS) introduced by Hartoog et al. (1973) to supplant the Russell-Bowen (1929) formula. These surveys found no evidence for Pm II, and lent very weak support to the original identification in HR 465.

In the present paper, we reopen the question of the possible presence of unstable elements in the atmospheres of magnetic Ap stars. We focus on Pm, as will be explained below. A reinvestigation is now suitable. New UVES spectra obtained by SH at ESO of HD 101065 and HD 965 are superior to those for HR 465 in wavelength coverage as well as signal-to-noise and resolution. The modern analytical tools are also superior to those used in 1960. Nevertheless, the putative Pm II lines are not strong in the spectrum, and our arguments for its presence are based on statistical tools and decades of experience in conventional line-identification work.

The presence of Pm II in either of these stars would mean that an unrecognized mechanism must exist to modify the surface chemistry. Prior to 1970, surface nuclear reactions would have been an obvious possibility. They have been largely ignored since the early 1970's, with the exception of solar flares, where there is now a large literature describing $\gamma$-rays and various nuclear reactions (Ramaty et al. 1995).

\section{Observations and line identifications - HD 101065; Pm in HD 965}

Most of the wavelengths analyzed here are from Cowley et al. (2000). They were measured from spectra of HD $101065 \mathrm{ob}-$ tained by GM using the ESO NTT and EMMI spectrograph. The epoch is 7 February, 1998. These were supplemented by measurements of UVES spectra obtained by SH as well as from the ESO archive. The $S / N$ is 200 or better, and the resolution is 80000 or above for all material. Each wavelength is accompanied by a measurement of a central intensity if applicable, or a visual estimate of this intensity in the case of severe blending. All wavelength measurements and identifications ${ }^{1}$ are posted. Our WCS studies were based on 11916 stellar lines from 3047 to $6822 \AA$.

Line identifications were made by WPB using traditional techniques. Many identifications with second spectra of rare earth elements (REE) were made automatically, by writing in all features in NBS Monograph 145 (Meggers et al. 1975) for Ce II, Nd II, Pr II, Sm II, Gd II, Dy II, and Er II that coincided to within $0.03 \AA$ with the stellar positions. The automatic identifications were reviewed and supplemented, with additional contributors, by WPB.

Our attention was originally drawn to the possibility that unstable elements might be present in the atmosphere of HD 101065 from stellar measurements close to strong Tc II lines in the violet.

In the discussion that follows, we shall use an asterisk to indicate a measured wavelength available at the URL given above. Thus, for example, $\lambda^{*} 3298.83$ indicates a measured stellar wavelength, while $\lambda 3298.84$ is the laboratory position,

\footnotetext{
${ }^{1}$ http://www.astro.1sa.umich.edu/users/cowley/
}

usually from Reader \& Corliss (1980), or Sansonetti \& Martin (2003).

The deep ultraviolet wavelength measurements contain $\lambda \lambda^{*} 3298.83$, 3892.05, and 3975.02, close to Tc II lines. Three additional Tc II lines at $\lambda \lambda 3195.20,3212.02$, and 3237.02 were then sought on ESO archival material. Only one of the three lines had a close match $\left(\lambda^{*} 3212.06\right)$. It may still be possible to establish the presence of Tc II in HD 101065; this task is left for future work. Space observations are critical for Tc II, whose resonance lines lie at $\lambda \lambda 2543,2610$, and 2647.

Interestingly, the case for the presence of Tc I seems better than that for Tc II. This is our conclusion both from the traditional technique used by WPB and the wavelength coincidence statistics (see below). We obtain "significances" (97 and 98\% confidences) with a list of laboratory wavelengths from Bozman et al. (1968) intensities of 5000 or greater. These are obtained for wavelengths within 10 and $20 \mathrm{~m} \AA$ respectively. A shorter list of lines from NIST (Sansonetti \& Martin 2003) gives no significance at any of the four tolerances tried $(10,20$, 40 , and $60 \mathrm{~m} \AA$ ).

Evidence for the presence of technetium is marginal, and we will not discuss it further in this paper. Because of the possible presence of technetium, we made extended surveys for many elements in HD 101065 using WCS, but with four tolerances, rather than our usual $0.06 \AA$, and several laboratory lists. These results gave apparently positive results for $\mathrm{Pm}$. A marginally positive result had been found for Pm II in a survey by WCS of wavelengths of HD 965 in 2000, but was dismissed. However when the HD 965 wavelengths were analyzed by WCS using additional lists and tolerances, the Pm results could no longer be ignored.

\section{WCS studies in HD 101065}

Hartoog et al. (1973) described the WCS method. Briefly, coincidences (or hits, $H$ ) with a list of $N$ laboratory lines is compared with the number of hits $\left(H_{\mathrm{R}}\right)$ on 5000 sets of nonsense wavelengths constructed by adding a random wavelength increment to the laboratory lines. In this way, we construct the Monte Carlo probability that the $H$ hits are due to chance from the fraction of hits $H_{\mathrm{R}}$ that are equal to or greater than $H$. We report this probability as a significance (SIG) with possible values from 0 to 1 . A low value of SIG (see tables below) means a small probability the coincidences are due to chance. The tolerance windows for these tests were $0.01,0.02,0.04$, and $0.06 \AA$.

Those unfamiliar with WCS often think that virtually any identification can be made in a spectrum with a very high line density. In fact, the opposite is true because a high density increases the number of coincidences with the nonsense wavelengths. Indeed, we find the highest significances for the promethium identification from the yellow and red regions of the spectrum where the line density is lower than in the blue and violet. lines.

We present results for WCS tests for the following sets of

1. All (152) lines from the NIST site (Sansonetti \& Martin 2003) for "strong" Pm I and II lines (Pm12 in Table 1); 
Table 1. Statistics of wavelength coincidences for HD 101065.

\begin{tabular}{|c|c|c|c|c|c|c|c|c|c|c|}
\hline & \multirow[b]{2}{*}{ ION } & \multirow[b]{2}{*}{ LINES } & \multicolumn{2}{|c|}{$10 \mathrm{~m} \AA$} & \multicolumn{2}{|c|}{$20 \mathrm{~m} \AA$} & \multicolumn{2}{|c|}{$40 \mathrm{~m} \AA$} & \multicolumn{2}{|c|}{$60 \mathrm{~m} \AA$} \\
\hline & & & HITS & SIG & HITS & SIG & HITS & SIG & HITS & SIG \\
\hline \multirow{3}{*}{$\begin{array}{l}11916 \text { stellar lines } \\
(3047-6822 \AA)\end{array}$} & Pm12 & 151 & 20 & 0.079 & 35 & $! 0.013$ & 54 & $! 0.030$ & 71 & 0.064 \\
\hline & $\mathrm{Pm} 2$ & 62 & 10 & 0.123 & 17 & !0.035 & 25 & 0.104 & 29 & 0.500 \\
\hline & $\mathrm{PM} 2 \mathrm{w}$ & 229 & 31 & 0.093 & 55 & $! 0.010$ & 79 & 0.207 & 104 & 0.530 \\
\hline \multirow{3}{*}{$\begin{array}{l}4852 \text { stellar lines } \\
(4949-6822 \AA)\end{array}$} & Pm12 & 55 & 11 & $! ! 0.003$ & 17 & !!0.001 & 24 & !!0.001 & 27 & !! 0.008 \\
\hline & Pm 2 & 16 & 4 & 0.052 & 6 & $! 0.026$ & 10 & $!$ !0.003 & 12 & $! ! 0.004$ \\
\hline & $\mathrm{PM} 2 \mathrm{w}$ & 60 & 7 & 0.250 & 14 & $! 0.041$ & 19 & 0.168 & 29 & $! 0.037$ \\
\hline \multirow{3}{*}{$\begin{array}{l}11916 \text { displaced } \\
(+7 \AA, 3055-6829 \AA)\end{array}$} & Pm12 & 151 & 15 & 0.465 & 26 & 0.339 & 40 & 0.736 & 54 & 0.915 \\
\hline & $\operatorname{Pm} 2$ & 62 & 4 & 0.908 & 8 & 0.888 & 14 & 0.966 & 21 & 0.982 \\
\hline & $\mathrm{PM} 2 \mathrm{w}$ & 229 & 25 & 0.479 & 38 & 0.696 & 68 & 0.782 & 97 & 0.848 \\
\hline \multirow{3}{*}{$\begin{array}{l}4852 \text { displaced } \\
(+5 \AA, 4954-6827 \AA)\end{array}$} & Pm12 & 54 & 9 & $! 0.023$ & 12 & $! 0.036$ & 14 & 0.351 & 19 & 0.401 \\
\hline & $\operatorname{Pm} 2$ & 16 & 3 & 0.180 & 4 & 0.227 & 5 & 0.467 & 5 & 0.819 \\
\hline & $\mathrm{PM} 2 \mathrm{w}$ & 60 & 8 & 0.134 & 13 & 0.073 & 17 & 0.340 & 24 & 0.327 \\
\hline \multirow{3}{*}{$\begin{array}{l}11916 \text { displaced } \\
(+12 \AA, 3059-6834 \AA)\end{array}$} & $\operatorname{Pm} 12$ & 151 & 13 & 0.698 & 22 & 0.717 & 45 & 0.406 & 65 & 0.334 \\
\hline & $\operatorname{Pm} 2$ & 62 & 6 & 0.676 & 10 & 0.703 & 22 & 0.353 & 28 & 0.639 \\
\hline & $\mathrm{PM} 2 \mathrm{w}$ & 229 & 18 & 0.941 & 36 & 0.816 & 70 & 0.702 & 94 & 0.928 \\
\hline \multirow{3}{*}{$\begin{array}{l}4852 \text { displaced } \\
(+10 \AA, 4959-6832 \AA)\end{array}$} & Pm12 & 54 & 4 & 0.574 & 6 & 0.677 & 10 & 0.803 & 15 & 0.812 \\
\hline & $\operatorname{Pm} 2$ & 16 & 1 & 0.793 & 1 & 0.937 & 1 & 0.995 & 4 & 0.939 \\
\hline & $\mathrm{PM} 2 \mathrm{w}$ & 60 & 7 & 0.248 & 10 & 0.336 & 16 & 0.450 & 26 & 0.158 \\
\hline
\end{tabular}

2. all (62) Pm II lines from the above set (Pm 2 in Table 1);

3. a set of (229) lines from Meggers et al. (1951) with lines in the intensity range 5 to 30 (PM2w in Table 1).

It is important to note that the second set of lines is a subset of both the first and third lists. However, the lines in the first set that are not in the second are all classified as Pm I. Of the 229 lines of the third set, all but 31 may be classified as Pm II from material on the NIST web sites. These 31 lines cannot be classified as either Pm I or II from the NIST sites, though they probably belong to one spectrum or the other.

The third set, PM2w, was made up in an attempt to reconstruct the list of lines used by Hartoog, et al. for which they found marginal significance for the coincidences in HR 465 (see their Table 3). The original list was on punched cards, and has been lost.

In addition to tests with the full 11916 measured lines, WCS were compiled for a subset of 4852 lines with wavelengths greater than $4949 \AA$. The longer wavelengths have markedly less blending, and offer some advantages over the full set. Pm II, like most of the lanthanides, has its strong lines in the violet. Thus the longer wavelength region gains due to reduced line blending, but does not contain the intrinsically strongest lines of the ion. Nevertheless, the best case for the presence of $\mathrm{Pm} \mathrm{II} \mathrm{is} \mathrm{from} \mathrm{the} \mathrm{longer-wavelength} \mathrm{region} \mathrm{of} \mathrm{the}$ spectrum.

The situation with Pm II is reminiscent of the historical debate over the presence of Fe I in this star. Przybylski (1977) maintained Fe I was very weak or absent altogether in HD 101065. Wegner \& Petford (1974), however, had measured equivalent widths of many Fe I lines in the visual region of the spectrum, and determined an essentially normal iron abundance. The situation remained moot until measurements of the ESO material and WCS analysis proved Fe I present at ineluctable confidence levels (Cowley et al. 2000).

Indeed, the assertion that a given atomic or ionic spectrum is very weakly present in some stellar spectrum is virtually non-falsifiable. This is a drawback of the traditional method of identifications. One must use it, but successful usage is not easy. There are cases where the presence of an atomic spectrum is clear and there could be no argument. But as one examines spectra that are only weakly represented, experience and subjective factors enter strongly. There is no rigorous way to duplicate and therefore confirm a subjective judgement. With WCS, one can at least duplicate the numerical results, even though the ultimate judgements must be based on probabilities.

Table 1 gives the results of the WCS tests for Pm II. The first section of the table (above the double lines) pertains to the stellar measurements. The column LINES gives the number of laboratory lines sought, while the four HITS columns give the number of coincidences within the four tolerances, 10, 20, 40, and $60 \mathrm{~m} \AA$. Coincidences found significant (SIG) at the 0.050 to 0.010 (95 to $99 \%$ confidence) level are marked with a single exclamation mark (!). Higher confidences are indicated by double exclamation marks (!!). In addition to the full set of stellar wavelengths and the subset containing only the visual 
region, results are also shown for control experiments, consisting of the stellar wavelengths displaced by an integral number of angstroms as indicated in the table. These entries serve as a second control on the experiment. We have no reason to think that one set of "fake" wavelengths is any more satisfactory as a control than any other. The ones displayed here were the first for which WCS were carried out on nonsense stellar data. The shifts were different for the full sets and the subsets in the visual.

The two significant results that occur for the control sets, at levels 0.023 and 0.036 , would not be improbable in the 48 independent experiments represented in the second part (Sects. 3-6) of Table 1. Actually the experiments are not independent. The wavelength lists overlap, and hits at $\pm 0.01 \AA$ are contained in the hits at $\pm 0.02 \AA$, etc.

In the case of the actual stellar wavelengths, even one of the 0.001 results is unexpected. In $4 \times 3=12$ random experiments we could expect one result at the $1 / 12=0.083$ significance level. In fact, there are 6 results at the 0.01 significance level or better.

Clearly, there are many more coincidences of stellar and promethium wavelengths than would occur by chance. One trivial explanation would be contamination of the laboratory wavelengths with other lanthanide lines. We discuss this possibility in a later section.

While the individual entries for each list and tolerance provide significances, what conclusions may be drawn from the table as a whole? We attempt an answer for the case of the 4852 line set. We form the sum of the 12 entries (SIG), which is 0.591 . We then perform 200 experiments with nonsense stellar wavelengths, formed by adding to or subtracting from the actual stellar wavelengths. We used half-integral values of an angstrom in the range 1.5 to $50.5 \AA$. For each experiment, we add the 12 entries. Since each entry can in principle range from zero to unity, the minimum value for the sum of the entries is zero, and the maximum is 12 . Figure 1 shows a histogram of the distribution of the 200 sums, $x$, along with an analytical fit in which the distribution is modeled as a beta function:

$f(x)=A x^{a}(12-x)^{b}$.

We find $a=2.088941$, and $b=1.544707$ by matching the empirical mean and variance of the 200 outcomes. The normalization constant $A=2.176429 \times 10^{-4}$, for the integral of $f(x)$ between 0 and 12 to be unity. For the plot, $f(x)$ has been multiplied by 400 , to conform with the binning and number of trials. In the 200 trials, the smallest three values obtained for the sum were respectively $0.699,1.340$, and 1.484 . Thus the significance of the value 0.591 obtained with the measured stellar wavelengths may be judged to be smaller than 0.005 ( 1 in 200). The integral of the function above, from 0 to 0.6 (we round the 0.591 ) is 0.000637 ( 1 in 1570). However, the integral from 0 to 0.7 (we round the 0.699 ) is only 0.00105 (or 1 in 952). Since we obtained 0.699 for one of the sums in 200 trials, it appears the formula may miss the significance by a factor of $952 / 200=4.8$, though we consider this reasoning to be conservative. We can multiply the value for the formula for the significance for 0.6 by 4.8 to obtain 0.00306 , which is just a little smaller than $1 / 200$. It seems reasonable to assume that the
Histogram of sums

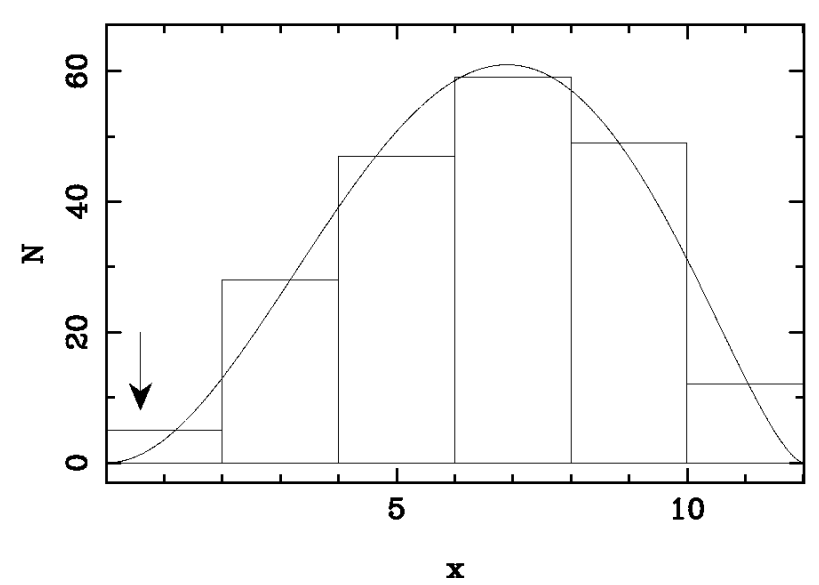

Fig. 1. Histogram of the probability sums for 12 trials ( 3 lists and 4 tolerances) based on 200 off-wavelength sets of 4852 wavelengths. The value of $x$ for the on-wavelength trials was 0.591 , and is indicated by the arrow. None of the 200 trials gave a value that small, but one trial gave 0.699 .

significance of the coincidences with the Pm lines used is at the level of from 1 in 200 to perhaps 1 in 300.

\section{Individual lines in HD 101065}

A line-by-line comparison of expected strong laboratory lines with stellar features is fraught with the opportunity for prejudiced judgements. This is especially true in the violet, where blending is severe. Consider the seven persistent, classified Pm II lines in the NIST Handbook below 15000: 3910.26, 3936.48, 3998.96, 4075.84, 4086.10, 4297.78, and 4417.96. Of these only 3 have stellar wavelengths within $0.06 \AA$ in our original measurements. New measurements using less severely smoothed spectra reveal 5 stellar features within $0.06 \AA$, and two coincide within $0.02 \AA$. Our measurement accuracy is surely of the order of $0.02 \AA$, but blends may easily perturb or obliterate a wavelength over a wider interval. The stellar feature at $\lambda^{*} 4297.79$ is identified on our website with strong lines of Pr II and Nd II. The line $\lambda^{*} 3910.28$ could be Ho I $\lambda 3910.30$.

The presence of first spectra of the lanthanides is an intriguing subject. Cowley et al. (2000) felt the first spectra were not securely identified. The case is much stronger with our additional material. Note that the Pm12 list appears to give slightly more significant results than its subset, Pm 2 . This could be a statistical fluke, an indication of some Pm II lines among the Pm I laboratory list, which Dr. Reader thinks very improbable, or a result of Pm I also being present in HD 101065. The discussion of this short list of lines has carried us somewhat afield, but is typical of the attempt to establish the presence of a weakly represented atomic or ionic spectrum within a rich and blended stellar spectrum.

The worst case among the seven classified lines is 24086.10. It falls some 0.04 to $0.05 \AA$ from a local intensity maximum. However, the maximum is still 10 to $15 \%$ deep, depending on the (uncertain) location of a continuum. Without careful study, this line cannot yet be used to exclude the weak 
presence of Pm II. The line has hyperfine structure $0.11 \AA$ aide. Beyond $5000 \AA$, line clutter in HD 101065 is far less severe, as noted long ago by Wegner and Petford. This is the reason for the separate WCS runs using only visual wavelengths. The region also provides the best opportunity to observe nearly unblended Pm II features. There are 16 lines of Pm II among the strong Pm I and II lines in the NIST Handbook. As can be seen from the second section of Table 1, an improbable (compared to chance) number of coincidences with these lines was found at all four tolerances, if we include the 4 coincidences at $\pm 0.01 \AA$ which are significant at 0.052 , just below our arbitrary threshold for an exclamation point.

The Pm II lines at $\lambda \lambda 6659.05$ and 6772.29 were both beyond the range measured for the 2000 study. Newer measurements revealed features which were measured at $\lambda \lambda^{*} 6659.07$ and 6772.28 . The spectrum was measured prior to finding the radial velocity shift, so neither line was recognized during the measurement. These two lines have equivalent widths of roughly 25 and $15 \mathrm{~m} \AA$, respectively. Calculations based on the 2000 model predict a Ni I line at 6772.32 , but it would be only $2 \%$ deep, while the putative Pm II feature is some 10\% deep.

Two Pm II lines with unknown energy levels were not measured, and fall near regions close to the continuum. Oscillator strengths, level classifications, and high $S / N$ observations near $\lambda \lambda 5153.86$ and 5246.33 could exclude the Pm II identification.

\section{HD 965}

Wavelength measurements were made by CRC and DJB on ESO CASPEC material obtained by GM on December 2, 1997. The instrument is described by Mathys \& Hubrig (1997). The resolution of the spectra is 35000 or higher, and $S / N 110$ to 150 . Measurements from 5530 to $6794 \AA$ are available ${ }^{2}$ on line. WCS tests were run at the four tolerances for the three wavelength lists discussed for Przybylski's star. The results for 2296 stellar lines are shown in the first part of Table 2. In the bottom part of the table, we show statistics based on more recent UVES spectra obtained by SH on 17 September 2002.

The UVES spectra are of higher quality than the CASPEC spectra, with resolutions of 80000 in the blue-violet, and 110000 beyond $4600 \AA$; the $S / N$ is $150-200$ in the violet, and more than 300 at longer wavelengths. It is unclear why the WCS results are less impressive than with the older material. Possibly the explanation is simply the higher line density of the newer list. The spectrum is definitely variable, and this is also a possible explanation.

There is no avoiding the very high significance of the coincidences with the CASPEC measurements. The results are significant for most tolerances for all three lists, and in some cases the level of significance is very high. For example, at a tolerance of $20 \mathrm{~m} \AA$, the number of coincidences on the random wavelengths averaged 3.24 with a standard deviation estimate (square-root of the variance) of 1.72. For Gaussian statistics, 14 HITS would indicate a $6.2 \sigma$ result. However, this is clearly an overestimate of the significance, since we have made tests

\footnotetext{
2 http://www.astro.1sa.umich.edu/users/cowley/ hd965wl.html
}

at 4 tolerances for 3 laboratory lists. In addition, we have not shown the results for another 12 trials with the $3 \mathrm{Pm}$ lists using the full measurements of 9960 (UVES) spectral lines from 3306 to $6663 \AA$.

Perhaps a better measure of the overall significance of the Table 2 entries is the sum of the significance (SIG). As discussed above, this could be a number between 0 and 12 . We made a similar Monte Carlo estimate for the distribution function of this sum using 200 displaced sets of the 2296 measured lines. The distribution can again be modelled as a beta function (see Eq. (1) above). We find $a=2.17822, b=0.818840$, and the normalization $A=5.22520 \times 10^{-4}$. This distribution is skewed toward large values of $\mathrm{x}$ somewhat more than that shown in Fig. 1; the maximum, mean, and mode are all between 7.5 and 8.0. The smallest sum of the 12 SIG's for the 200 Monte Carlo trials was 2.047 . The probability of the sum being as small as 0.14 (first section of Table 2) would be $2.4 \times 10^{-6}$ according to this model.

\section{Contamination or nature's perversity}

We thank Dr. Joseph Reader of NIST for his opinion that contamination of the promethium laboratory lists by other atomic or ionic lines can be ruled out. At the suggestion of the referee, Dr. John Landstreet, we searched specifically for evidence of contamination by $\mathrm{Nd}$ and $\mathrm{Sm}$ in the laboratory lists described in Sect. 3. We actually found an improbable lack of chance coincidences of the Pm I and II lines with laboratory lines of these elements. Possibly the laboratory spectroscopists had been overly conservative in purging possible contaminants from these elements from the Pm lists.

We performed additional tests of our own, and conclude that possibly relevant correlations of the promethium wavelengths with lines from stable elements do indeed exist. Whether this is from contamination of the laboratory line list or the perversity of nature remains to be seen. What is important is whether our analysis of the stellar material is influenced by these correlations.

We have tested the three promethium lists of Sect. 3 against three wavelength lists constructed from NBS Monograph 145 (Meggers et al. 1975), and NBS Monograph 61 (Moore et al. 1966). The wavelength ranges and number of lines were chosen to be similar to those of the visual-region tests of HD 101065 and HD 965. The laboratory (Mon. 145) and solar lines have been treated in the same way as stellar wavelengths, and the statistical summary of Table 3 has essentially the same structure as the previous tables. Some lists were "thinned" using a random number to decide whether to keep or discard a line.

The lists are as follows:

1. all Mono. 145 lines of the second spectra of lanthanide rare earths, uranium and thorium, plus randomly chosen first spectra to increase the line density: 3598 lines in the domain 5000-8958 A;

2. the above list, but with lines randomly deleted, to yield a set of 2270 lines with a line density near that of HD 965;

3. all lines from the first spectra of all elements in Mono. 145: 4762 lines in 4950-6780 ̊̊; 
Table 2. Statistics of wavelength coincidences for HD 965.

\begin{tabular}{|c|c|c|c|c|c|c|c|c|c|c|}
\hline & \multirow[b]{2}{*}{ ION } & \multirow[b]{2}{*}{ LINES } & \multicolumn{2}{|c|}{$10 \mathrm{~mA}$} & \multicolumn{2}{|c|}{$20 \mathrm{~m} \AA$} & \multicolumn{2}{|c|}{$40 \mathrm{~m} \AA$} & \multicolumn{2}{|c|}{$60 \mathrm{~mA}$} \\
\hline & & & HITS & SIG & HITS & SIG & HITS & SIG & HITS & SIG \\
\hline \multirow{3}{*}{$\begin{array}{l}2296 \text { stellar lines } \\
\text { (5531-6795 ̊) }\end{array}$} & $\mathrm{Pm} 12$ & 39 & 8 & !!0.001 & 14 & !!0.000 & 16 & !!0.000 & 18 & !!0.001 \\
\hline & Pm 2 & 7 & 3 & !!0.004 & 5 & $! ! 0.000$ & 6 & !!0.000 & 6 & !!0.001 \\
\hline & $\mathrm{PM} 2 \mathrm{w}$ & 18 & 3 & 0.069 & 55 & !0.010 & 7 & !0.016 & 8 & $! 0.038$ \\
\hline \multirow{3}{*}{$\begin{array}{l}4388 \text { stellar lines } \\
\text { (4952-6662 ̊) }\end{array}$} & $\mathrm{Pm} 12$ & 39 & 10 & !!0.001 & 13 & !!0.000 & 18 & !!0.002 & 23 & !!0.001 \\
\hline & $\operatorname{Pm} 2$ & 14 & 3 & 0.323 & 6 & !0.026 & 14 & 0.279 & 9 & !0.033 \\
\hline & $\mathrm{PM} 2 \mathrm{~W}$ & 55 & 8 & 0.499 & 14 & $! 0.041$ & 15 & 0.394 & 22 & 0.304 \\
\hline
\end{tabular}

Table 3. Statistics of Pm coincidences with solar and laboratory wavelengths.

\begin{tabular}{|c|c|c|c|c|c|c|c|c|c|c|}
\hline & \multirow[b]{2}{*}{ ION } & \multirow[b]{2}{*}{ LINES } & \multicolumn{2}{|c|}{$10 \mathrm{~m} \AA$} & \multicolumn{2}{|c|}{$20 \mathrm{~mA}$} & \multicolumn{2}{|c|}{$40 \mathrm{m \AA}$} & \multicolumn{2}{|c|}{$60 \mathrm{~m} \AA$} \\
\hline & & & HITS & SIG & HITS & SIG & HITS & SIG & HITS & SIG \\
\hline \multirow{3}{*}{$\begin{array}{l}3598 \text { NBS, mostly } \\
\text { second REE spectra }\end{array}$} & Pm12 & 54 & 3 & 0.405 & 5 & 0.325 & 11 & 0.055 & 17 & $! ! 0.005$ \\
\hline & Pm 2 & 16 & 1 & 0.633 & 2 & 0.470 & 3 & 0.520 & 5 & 0.312 \\
\hline & $\mathrm{PM} 2 \mathrm{w}$ & 57 & 3 & 0.630 & 6 & 0.432 & 9 & 0.594 & 8 & 0.300 \\
\hline \multirow{3}{*}{$\begin{array}{l}2270 \text { NBS,mostly } \\
\text { second REE spectra }\end{array}$} & Pm12 & 39 & 3 & 0.174 & 4 & 0.208 & 8 & $! 0.049$ & 13 & !!0.004 \\
\hline & $\operatorname{Pm} 2$ & 14 & 1 & 0.466 & 2 & 0.269 & 3 & 0.249 & 5 & 0.093 \\
\hline & $\mathrm{PM} 2 \mathrm{w}$ & 55 & 1 & 0.869 & 2 & 0.848 & 4 & 0.851 & 9 & 0.439 \\
\hline \multirow{3}{*}{$\begin{array}{l}4762 \text { NBS, } \\
\text { all first spectra }\end{array}$} & Pm12 & 53 & 5 & 0.255 & 8 & 0.184 & 16 & $! 0.015$ & 19 & !0.039 \\
\hline & $\operatorname{Pm} 2$ & 15 & 3 & 0.135 & 5 & $! 0.047$ & 8 & $! 0.014$ & 9 & $! 0.024$ \\
\hline & $\mathrm{PM} 2 \mathrm{w}$ & 58 & 11 & $! 0.013$ & 16 & !!0.006 & 21 & !0.031 & 24 & 0.119 \\
\hline \multirow{3}{*}{$\begin{array}{l}2334 \text { NBS, } \\
\text { all first spectra }\end{array}$} & Pm12 & 53 & 4 & 0.101 & 5 & 0.158 & 8 & 0.130 & 12 & $! 0.045$ \\
\hline & $\operatorname{Pm} 2$ & 15 & 2 & 0.147 & 2 & 0.311 & 3 & 0.310 & 5 & 0.122 \\
\hline & $\mathrm{PM} 2 \mathrm{w}$ & 58 & 9 & !!0.002 & 12 & !!0.002 & 13 & 0.034 & 17 & $! 0.028$ \\
\hline \multirow{3}{*}{4985 solar lines } & $\operatorname{Pm} 12$ & 54 & 6 & 0.168 & 9 & 0.163 & 15 & 0.134 & 23 & $! 0.029$ \\
\hline & $\operatorname{Pm} 2$ & 16 & 3 & 0.168 & 4 & 0.216 & 7 & 0.100 & 11 & $! 0.008$ \\
\hline & $\mathrm{PM} 2 \mathrm{w}$ & 59 & 9 & 0.116 & 13 & 0.142 & 21 & 0.143 & 29 & 0.105 \\
\hline \multirow{3}{*}{2237 solar lines } & Pm12 & 54 & 1 & 0.827 & 2 & 0.797 & 3 & 0.910 & 6 & 0.780 \\
\hline & $\operatorname{Pm} 2$ & 16 & 0 & 1.000 & 0 & 1.000 & 0 & 1.000 & 1 & 0.957 \\
\hline & PM2w & 59 & 2 & 0.764 & 5 & 0.481 & 9 & 0.419 & 12 & 0.495 \\
\hline
\end{tabular}

4. randomly chosen lines from the previous list, selected so the line density would be comparable with that of the HD 965 list: 2334 lines in 4950-6778 ;

5. a list of 4985 lines randomly chosen from Mono. 61 in the range 4950-6816 $\AA$;

6. a list of 2337 lines randomly chosen from Mono. 61 in the range 4950-6815 ̊.

We have posted ${ }^{3}$ a list of the individual laboratory (Mono. 145) and solar wavelengths that coincide within $0.06 \AA$ of the Pm12 lines.

If the wavelengths from Monograph 61 and 145 had been stellar measurements, and the laboratory lists some noncontroversial spectra, we would consider the identification

\footnotetext{
${ }^{3}$ http://www.astro.lsa. umich.edu/users/cowley/ pm12bln.html
}

likely, but perhaps not beyond doubt. See, for example, the elements and confidence levels of Table 3 of Cowley et al. (2000). Thus, either the laboratory wavelengths are mildly contaminated, or an improbable, accidental correlation exists between the promethium wavelengths and the wavelengths of other atoms and/or ions.

\section{Concluding remarks}

The significance levels of coincidences of Pm I and Pm II lines with the stellar wavelengths of HD 101065 and especially for HD 965 are very high. Compare the results of Tables 1 (top portion) and 2 with those of Table 3 . In the former tables, the small values of SIG tend to persist across all tolerances and tend to be larger (lower confidence) for the largest tolerance 
(60 mÅ). By contrast, in Table 3, the smallest values of SIG occur more typically for the largest tolerance.

The spectroscopic evidence presented here would be more than adequate to justify asserting the presence of one of the stable lanthanides. Occasional missing stellar wavelength measurements of expected lines is characteristic of a spectrum that is weakly present - witness Przybylski's rejection of Fe I.

It is difficult to take account of the possible correlations of lines from stable elements with the promethium wavelengths (Sect. 6). Certainly it diminishes the confidence with which we announce a likely identification. Given Dr. Reader's opinion that the laboratory wavelengths were uncontaminated, we must invoke nature's perversity to explain Table 3. To go forward, and assert that the still higher confidences of Tables 1 and 2 are again due to chance is indeed a strain, though a most interesting one.

The traditional interpretation of HD 101065 and HD 965 is that they are cool CP stars, with deep radiative envelopes. This model provides no way to account for the presence of promethium in terms of mixing from the interior. Surface spallation was recently mentioned by Shavrina, et al. (2003), in connection with the $\mathrm{Li}-6 / \mathrm{Li}-7$ ratio. A similar surface process was suggested some decades ago by Tjin A Djie, Takens, and van den Heuvel (1973), where reactions of the form ${ }^{145} \mathrm{Nd}(\mathrm{p}, \mathrm{n}){ }^{145} \mathrm{Pm}$ or ${ }^{146} \mathrm{Nd}(\mathrm{p}, \mathrm{n}){ }^{146} \mathrm{Pm}$ could produce the evanescent nuclides from abundant isotopes with similar values of $A$.

It is most unlikely that confirmation of Pm in the spectra of these two highly peculiar stars would cause a reversion to the ideas of the 1960's (cf. Fowler et al. 1965), when peculiar A and B stars were thought to play a significant role in the galactic production of elements beyond the iron peak. We have already mentioned the plausibility of chemical separation as the major cause of abundance anomalies for upper main sequence stars. Thus, peculiar A and B stars are not currently thought to play a role in galactic chemical evolution. However, if the promethium identifications can be confirmed, there may well be some consequences for our ideas about galactic chemical evolution. Surface reactions could be relevant for special elements such as lithium, or nuclides, such as those attributed to the $p$-process.

In the present paper, we emphasize the spectroscopic evidence. Future work may reveal flaws in the current approach, or open an exciting new area of research for stellar astronomy.

Acknowledgements. We thank Drs. Charles Proffitt and Joseph Reader for comments on an early version of the present manuscript.
Comments by the referee, Dr. John Landstreet, improved the paper. We have used the VALD data base (Kupka et al. 1999), and are grateful for its availability. Some of the digitized wavelengths available on our web sites were prepared by Ms. Sheryl Smith.

\section{References}

Aller, M., \& Cowley, C. R. 1970, ApJ, 162, L145

Cowley, C. R. 1976, ApJS, 32, 631

Cowley, C. R. 1984, Phys. Scr., 8, 28

Cowley, C. R., Ryabchikova, T. A., Kupka, F., et al. 2000, MNRAS, 317, 299

Bozman, W. R., Corliss, C. H., \& Tech, J. L. 1968, J. Res. N.B.S., $72 \mathrm{~A}, 559$

Fowler, W. A., Burbidge, E. M., Burbidge, G. R., \& Hoyle, F. 1965, ApJ, 142, 423

Hartoog, M. R., Cowley, C. R., \& Cowley, A. P. 1973, ApJ, 182, 847

Hubrig, S., Cowley, C. R., Bagnulo, S., et al. 2002, in Exotic Stars as Challenges to Evolution, ed. C. A. Tout, \& W. Van Hamme, ASP Conf. Ser., 279, 365

Jaschek, C., \& Jaschek, M. 1995, The behavior of chemical elements in stars (Cambridge: University Press)

Kupka F., Piskunov N. E., Ryabchikova T. A., Stempels H. C., \& Weiss W. W. 1999, A\&AS, 138, 119

Mathys, G., \& Hubrig, S. 1997, A\&AS, 124, 475

Meggers, W. F., Corliss, C. H., \& Scribner, B. F. 1975, Tables of Spectral Line Intensities, 2nd ed. (Washington DC: US Gov. Print. Off.), Mono. 145

Meggers, W. F., Scribner, B. F., \& Bozman, W. R. 1951, J. Res. N.B.S., 46,85

Michaud, G. 1970, ApJ, 160, 641

Moore, C. E., Minnaert, M. G. J., \& Houtgast, J. 1966, The Solar Spectrum $2935 \AA$ to $8770 \AA$ (Washington DC: US Gov. Print. Off.), Mono. 61

Preston, G. W., \& Wolff, S. C. 1970, ApJ, 160, 1071

Przybylski, A. 1977, MNRAS, 178, 71

Ramaty, R., Mandzhavidze, N., \& Hua, X.-M. 1995, High Energy Solar Physics, AIP Conf. Proc., 374

Reader, J., \& Corliss, C. H. 1980, Wavelengths and Transition Probabilities for Atoms and Atomic Ions, Part I, NSRDS-NBS 68

Russell, H. N., \& Bowen, I. S. 1929, ApJ, 69, 196

Sansonetti, J. E., \& Martin, W. C. 2003, Handbook of Basic Atomic Spectroscopic Data, http://physics.nist.gov/ PhysRefData/Handbook/index.html

Shavrina, A. V., Polosukhina, N. S., Pavlenko, Ya. V., et al. 2003 [arXiv: astro-ph/0307464], 26 Jul. preprint

Tjin A Djie, H. R. E., Takens, R. J., \& van den Heuvel, E. P. J. 1973, Astrophys. Lett., 13, 215

Wegner, G., \& Petford, A. D. 1974, MNRAS, 168, 557 